\title{
Qucs Frequency Domain Non-Linear Compact Modelling and Simulation of IC Spiral Inductors On Silicon
}

\author{
Mike Brinson \\ Centre for Communications Technology \\ London Metropolitan University \\ UK \\ Email: mbrin72043@yahoo.co.uk
}

\begin{abstract}
SPICE AC circuit simulation is fundamentally a small signal network analysis of linear or non-linear circuits operating at specified DC bias conditions, where the circuit component values are assumed not to be functions of $\mathrm{AC}$ input signal frequency. In the case of RF circuit simulation this assumption can give rise to significant modelling errors. With the recent improvements in General Public License (GPL) circuit simulators this situation is changing, particularly through the introduction of Frequency Dependent Equation-Defined Device (FEDD) models, non-linear current/voltage static and dynamic Equation-Defined Device (EDD) models and user controlled swept signal frequency simulation employing Harmonic Balance steady state analysis. The main purpose of this paper is to introduce a number of novel modelling and circuit simulation techniques that allow, and enhance, the construction of compact device models with embedded behavioural components whose non-linear properties are functions of $\mathrm{AC}$ input signal frequency. To demonstrate these new modelling techniques a compact model for a $10 \mathrm{GHz}$ band width spiral inductor integrated on silicon is introduced, its compact model presented, and finally its simulation performance compared with published measured device data.
\end{abstract}

Index Terms-Qucs, Qucs-S, Ngspice, Xyce, compact device modelling, Frequency Equation-Defined Devices (FEDD), Equation-Defined Devices (EDD), SPICE B type sources, Harmonic Balance simulation.

\section{INTRODUCTION}

SPICE AC circuit simulation is essentially a small signal network analysis of linear or non-linear circuits operating at specified DC bias conditions [1], where the bias voltages and currents are considered to be fixed in value and component values are assumed not to be functions of other circuit variables, like for example AC input signal frequency. In the case of RF circuit simulation this assumption can give rise to significant modelling errors. One physical process that causes such an error is the well known AC current skin effect [2], where the resistance of a metal conductor increases above its DC value at a rate proportional to the square root of the applied signal frequency. This effect can be particularly marked in circuits that operate in the RF and microwave regions of the electromagnetic spectrum. SPICE 3f5 cannot directly handle linear or non-linear behavioural device models whose properties are entirely or partially characterised by a physical effect that is a function of AC signal frequency. With the recent improvements in General Public License (GPL) circuit simulators this situation is changing, particularly through the introduction of Frequency Dependent EquationDefined Device (FEDD) models[3], non-linear current/voltage static and dynamic Equation-Defined Device (EDD) models [4] and user controlled swept signal frequency simulation employing Harmonic Balance steady state analysis. The Quite Universal Circuit Simulator (Qucs) [5] is a package that implements a number of important frequency dependent extensions. In this context Qucs is really a generic name for a group of circuit simulators and modelling software tools that have evolved from a single Qucs GPL package to become a loosely linked software suite with elements specifically written for RF, microwave and communication system simulation (available with QucsStudio [6], while linking Qucs with the Ngspice [7], Xyce [8] and SPICE OPUS [9] circuit simulators (available with Qucs-S [10]), while simultaneously providing Verilog-A and XSPICE Code Model modelling plus synthesis capabilities. The main purpose of this paper is to introduce a number of novel modelling and circuit simulation concepts that allow, and enhance, the construction of compact device models with embedded behavioural components whose properties are functions of AC input signal frequency. To demonstrate these new modelling techniques a compact model for a $10 \mathrm{GHz}$ band width spiral inductor integrated on silicon is introduced [11] [13], its compact model presented, and finally its simulation performance compared with published measured device data.

\section{RF AND MicRowAVE Single LAYER SPIRAL INDUCTOR CHARACTERISTICS}

A diagram showing the surface layout (a), a cross-section side view (b) and a lumped equivalent circuit (c) for a microwave IC spiral inductor on silicon is given in Fig.1. The proposed inductor is a single layer, four sided, two and a half turn component, constructed from a thin film metal strip, etched in a spiral geometry, on a silicon substrate . Each section of the inductor is insulated from other parts of the component by silicon dioxide. Part (b) of Fig. 1 depicts the cross-section side view of the inductor cut horizontally 

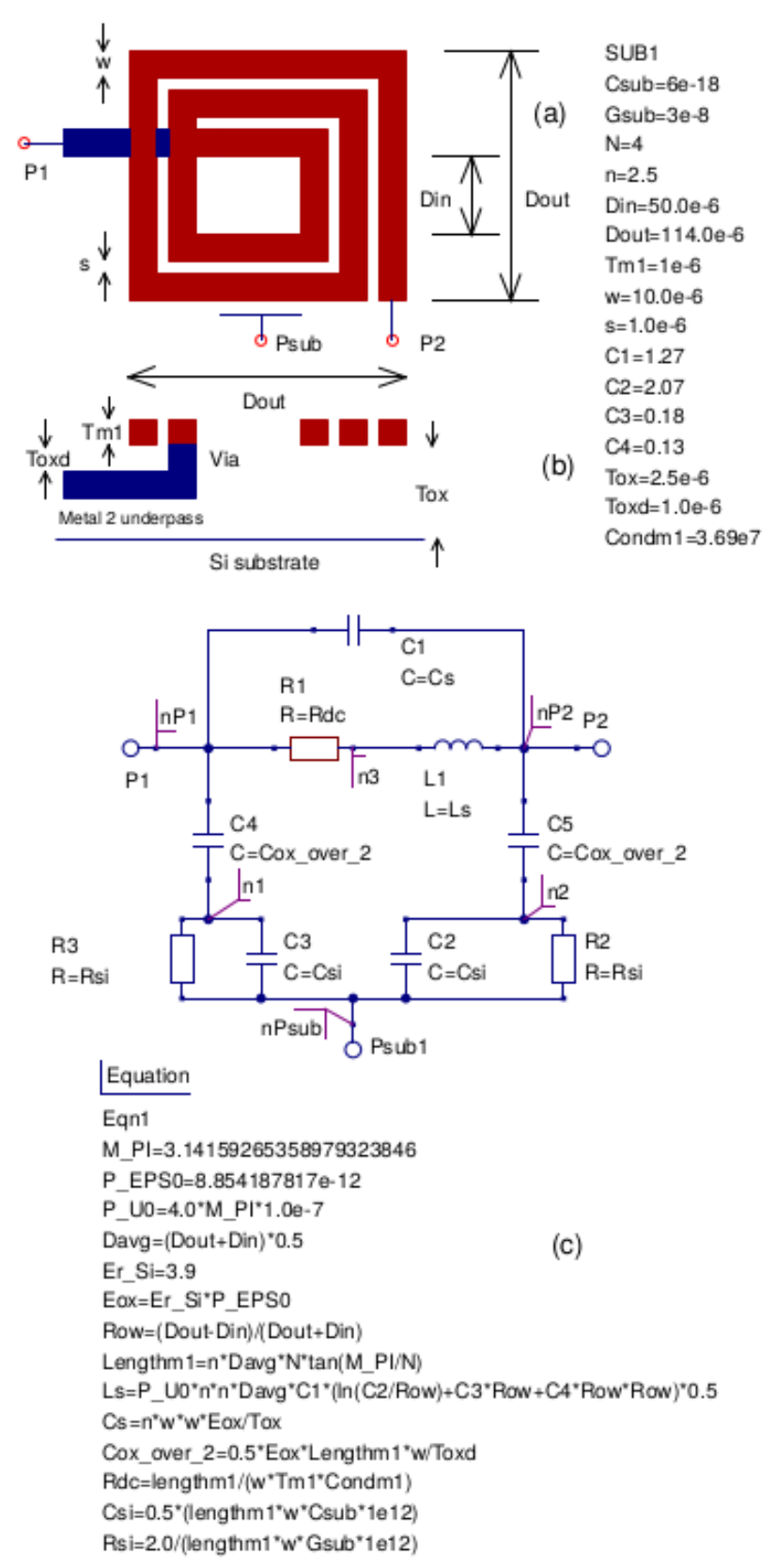

Fig. 1. Spiral inductor characteristics: (a) surface layout, (b) cross-section and (c) lumped equivalent electrical circuit.

through the component at a position that includes an underpass connection formed from a second metal layer. A low resistance "via" connects metal layers one and two, where metal layer one is the top metal layer. All dimensions listed in Fig.1 are in meters. For a lumped element model of the inductor to be valid at a given RF or microwave frequency, the inductor dimensions must be less than approximately one tenth of the signal wavelength. In Fig. 1

$$
P 1=\frac{P_{-} U 0 \cdot n^{2} \cdot D_{a v g} \cdot C 1}{2}
$$

$$
\begin{gathered}
\text { Ls }=P 1 \cdot\left(\ln \left(\frac{C 2}{R o w}\right)+C 3 \cdot \text { Row }+C 4 \cdot \text { Row }^{2}\right) \\
R d c=\frac{\text { Lengthm } 1}{w \cdot \text { Tm } 1 * \text { Condm } 1} \\
\text { Cs }=\frac{n \cdot w^{2} \cdot \text { Eox }}{\text { Toxd }} \\
\text { Cox_over_ } 2=\frac{\text { Eox } \cdot \text { Lengthm } 1 \cdot w}{\text { Tox }} \\
\text { Csi } \approx 0.5 \cdot \text { Csub } \cdot \text { Lengthm } 1 \cdot w \\
\text { Rsi } \approx \frac{2}{\text { Gsub Lengthm } 1 \cdot w}
\end{gathered}
$$

where

$$
\begin{aligned}
\text { Eox } & =\text { Er } r_{-} \text {Si } \cdot P_{-} \text {EPSO } \\
\text { Row } & =\frac{\text { Dout }- \text { Din }}{\text { Dout }+ \text { Din }} \\
\text { Davg } & =\frac{\text { Dout }- \text { Din }}{2} \\
\text { Lengthm } 1 & =n \cdot \text { Davg } \cdot N \cdot \tan \left(\frac{M_{-} P I}{N}\right)
\end{aligned}
$$

and expressions for each of the remaining right hand side named quantities in equations (2) to (5) are given in Fig. 1 (c) or Fig. 1 (a). Other undefined quantities are Gsub and Csub the conductance and capacitance per unit area of the silicon substrate respectively, $\operatorname{Tm} 1$ the thickness of metal layer one (in this model assumed to be $1 \mu \mathrm{m}$ thick aluminium), Condm 1 the electrical conductivity of metal one, and Lengthm 1 the length of inductor conductor $\mathrm{m} 1$. As expected the curve of Zinreal plotted against frequency, given in Fig. 2, indicates that Zinreal is roughly constant from low frequencies till nearly $10 \mathrm{GHz}$ due to the fact that this basic model does not include any non-linear resistive components that are functions of signal frequency.

\section{AdDING Frequency DEPENDENT Non-LineAR Resistive COMPONENTS TO THE SPIRAL INDUCTANCE MODEL}

Measurement of inductor series resistance at RF and microwave frequencies confirms that the resistive properties of a metal conductor change at high AC signal frequencies. Moreover, the rate of change is a strongly non-linear function of frequency. This effect is attributed to a combination of two effects; firstly conductor AC current skin effect [1] and secondly time dependent magnetically induced currents due to current crowding proximity effects, causing non-uniform, eddy like, current flow in the centre region of a conductor [14] and [17]. At DC, or low AC frequencies, inductor resistance $R d c$ is given by equation (3). Skin effect causes the DC resistance to increase at a rate proportional to the square root of frequency. Throughout the RF bands the skin effect is normally the dominant cause of increasing conductor resistance. At very high RF and microwave frequencies the inductance series resistance increases further becoming a complex function of both skin effect and magnetically induced 

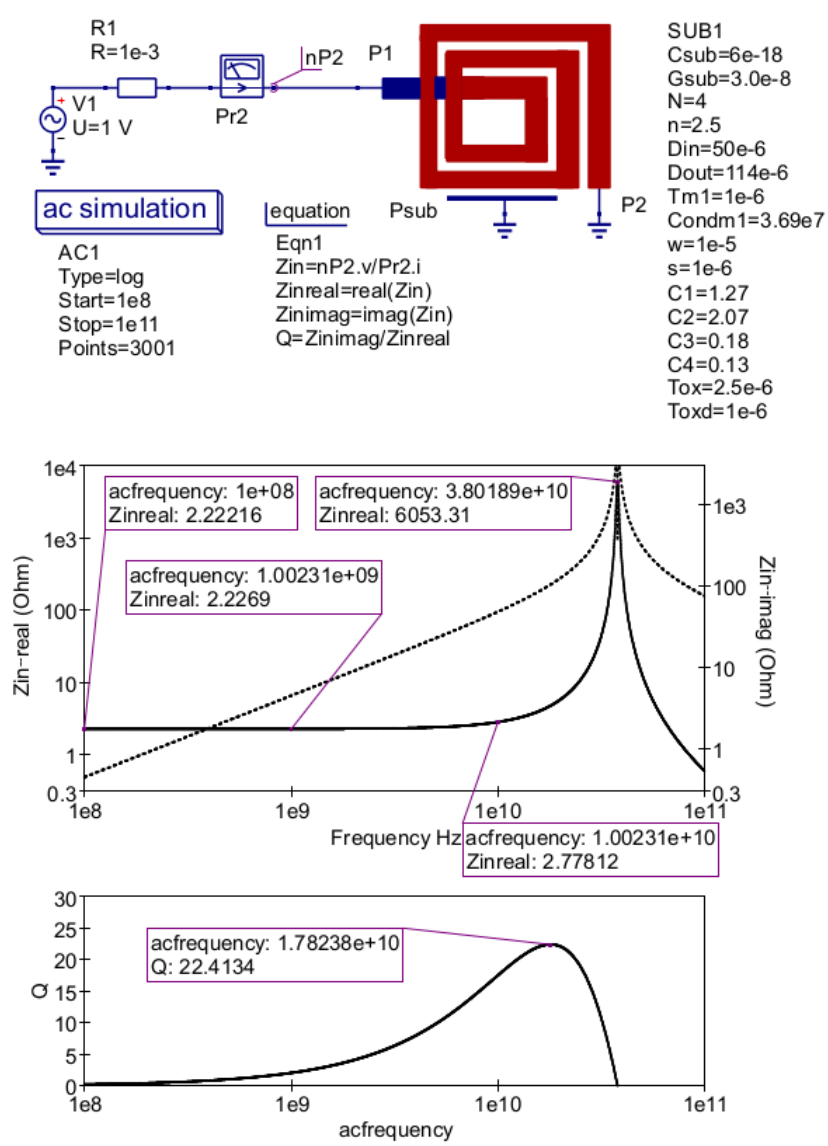

Fig. 2. A basic small signal AC test circuit for determining the real and imaginary components of inductance impedance and the component effective $\mathrm{Q}$ factor Zinimag/Zinreal.

currents. Measurements suggest that the additional increase in inductor resistance is proportional to the square of frequency. As a first approximation the inductor series resistance $R s$ can be expressed as

$$
R s(f)=R d c+R \operatorname{skin}(f)+R e d d y(f)
$$

where $R d c$ is the inductor series DC resistance (see Fig. 1 (c)), $R \operatorname{skin}(f)$ is the increase in conductor resistance due to skin effect and $\operatorname{Reddy}(f)$ is the increase in resistance due to the magnetic induced current crowding eddy effect. In this context

$$
\begin{gathered}
R \operatorname{skin}(f)=K 1 \cdot \sqrt{f} \\
\operatorname{Reddy}(f)=K 2 \cdot\left(\frac{f}{f c}\right)^{2}
\end{gathered}
$$

where

$$
f c=\frac{3.1 \cdot(w+s)}{2.0 \cdot \pi c \operatorname{dot} \mu_{0} \cdot w \cdot w} \cdot R s h
$$

Here, $R s h$ is the conductor sheet resistance in $\Omega \mathrm{m}$, and the $K 1$ and $K 2$ variables are real quantities that can be adjusted to fit the Rskin $(f)$ and Reddy equations to measured data. . Figure 3 illustrates how inductor series resistor $R s(f)$ is constructed from the DC value and the two frequency contributions to
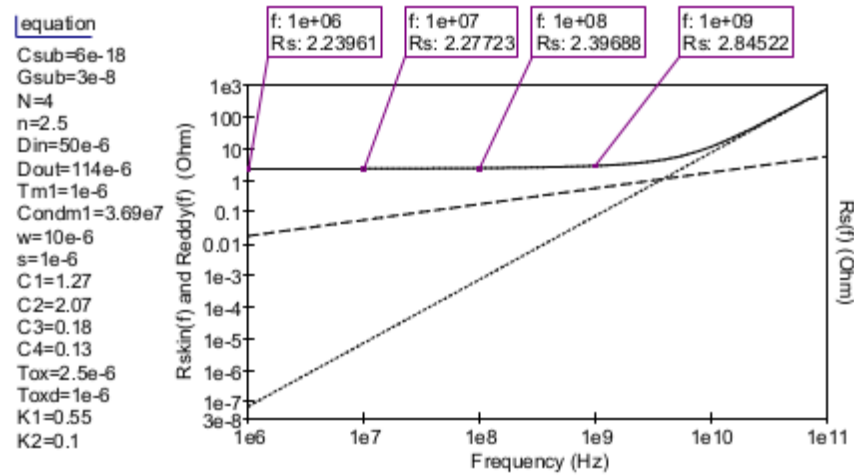

Fig. 3. A graph of $R s(f)$ against frequency for the model parameters listed at left hand side; Left scale dash curve $R \operatorname{skin}(f)$, dot curve Reddy $(f)$ and right scale black curve $R s(f)$.

$R s$. Without the two non-linear frequency contributions $R s=$ $R d c$. Hence, omission of the $R s$ frequency dependent terms can result in significant simulation errors at high frequency.

\section{SimUlation OF NON-LINEAR FREQUENCY DEPENDENT INDUCTOR MODELS}

\section{A. Introduction}

Simulation of non-linear frequency dependent device models can be very difficult with conventional SPICE circuit simulators [15]. Although the SPICE B non-linear independent source is part of the 3F5 software, in its original implementation voltage or current transfer functions cannot be written as algebraic expressions of signal frequency. Today a number of the circuit simulators forming the Qucs/Qucs-S/QucsStudio package have been extended to allow non-linear frequency dependent device models to be independent of, or combined with, the more usual static current/voltage and dynamic charge device models. These extended models include Frequency Dependent-Device models (FEDD), Equation-Defined Device models (EDD), and explicit frequency dependent SPICE B type models. At this time the latter being limited to Ngspice. When coupled with small signal AC and large signal Harmonic Balance simulation they provide users with a powerful set of extended modelling and simulation features.

\section{B. Qucs and QucsStudio non-linear AC circuit simulation with FEDD}

Both the original Qucs and the more recent QucsStudio circuit simulators include implementations of non-linear frequency dependent component models. These are constructed around standard two port electrical networks. Although the Qucs and QucsStudio simulators employ somewhat different netlists to encode a circuit under test, the differences are relatively minor. Hence, it is straightforward to use either simulator to investigate the performance of RF and microwave spiral inductors. Given in Fig. 4 is the equivalent circuit for a spiral inductor with an embedded FEDD that represents the series resistance $R s(f)$. In Fig. 4 resistor $R s(f)$ is shown 


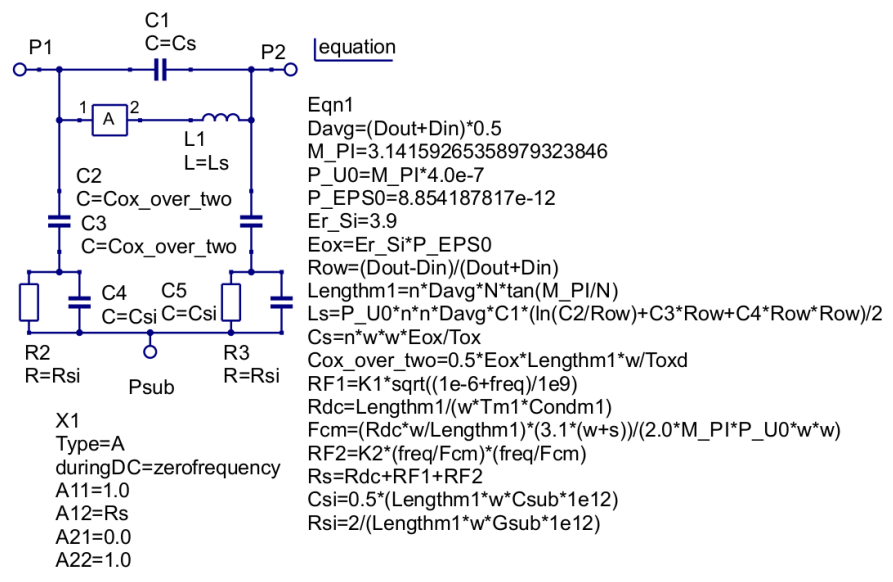

Fig. 4. An equivalent electric circuit for a spiral inductor with an embedded FEDD representing $R s(f)$.

modelled by an ABCD two port network. Both the Qucs and QucsStudio simulators include FEDD style models. Figure 5 illustrates the use of conventional small signal AC simulation to test the performance of the spiral inductance model specified in Fig. 4. Notice that the "ac simulation" icon is used to set the frequency for source $V 1$ and that the $\log$ frequency scan is controlled automatically by the circuit simulator.

\section{Qucs and QucsStudio non-linear HB circuit simulation with FEDD}

HB simulation is a relatively new addition to GPL circuit simulators. It has however, been implemented in the Qucs/QucsStudio and Xyce software packages. Both the QucsStudio and Xyce versions are more complete and stable than the original Qucs HB version. A number of other important differences also exist, namely that Qucs and QucsStudio allow FEDD style models to be included in a circuit under test, Xyce B style models do not allow frequency dependent terms in their $I$ or $V$ transfer functions, and finally Qucs and QucsStudio allow HB frequency to be changed with the Parameter sweep icon. Moreover, Xyce and Ngspice, or indeed other GPL versions of SPICE, do not normally allow the latter. The test circuit and plotted simulation data illustrated in Fig. 6, sections (a) to (c), suggest one use of "user controlled frequency Parameter sweeps". In this novel process parameter freq is changed in log or lin incremental steps and HB simulation undertaken after each change. The data plots in Fig. 6 (b) and (c) are illustrated, for clarity, with continuous connected lines rather than the more conventional $\mathrm{HB}$ arrow plot.

\section{INDIRECT NON-LINEAR FREQUENCY CIRCUIT SIMULATION USING EDD AND HARMONIC BALANCE.}

The Qucs/QucsStudio nonlinear EDD is well established as an advanced behavioural modelling component for representing device static $I(V)$ and dynamic $Q(I, V)$ properties. The Qucs-S package also implements EDD in the Ngspice and Xyce circuit simulators, via the Qucs-S GUI. Recent advances in Qucs and QucsStudio compact modelling and
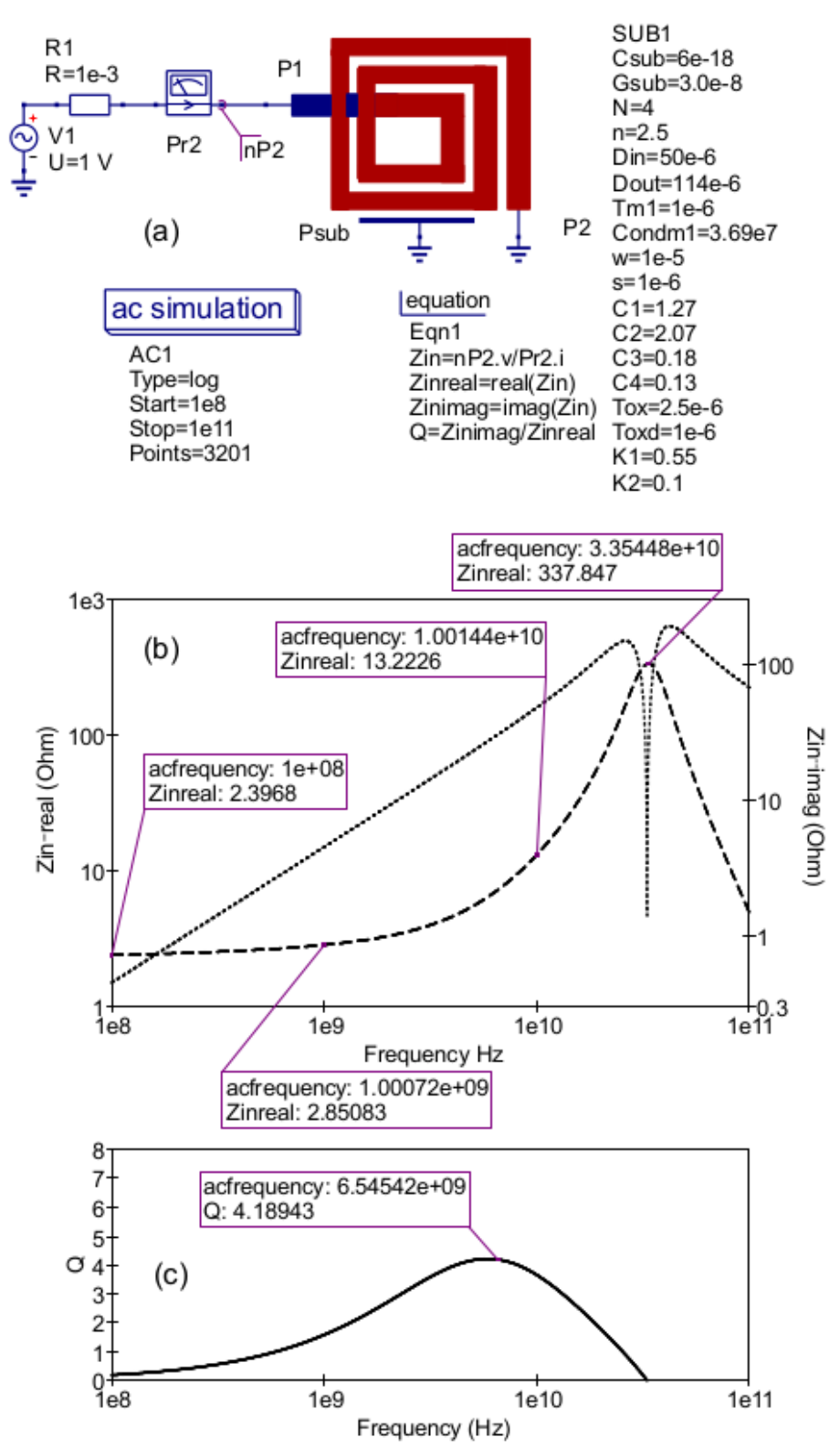

Fig. 5. Qucs and QucsStudio test bench and typical simulation output data with $R s(f)$ represented by an embedded FEDD: (a) small signal AC test circuit, (b) Zinreal (dash curve) and Zinimag $(f)$ (dot curve) plotted against frequency, and (c) Inductor effective $Q$ factor plotted against frequency.

simulation have shown that it is also possible to use EDD when constructing functions of the $R s(f)$ form. Shown in Fig. 7 is an extended equivalent circuit for the spiral inductor introduced in previous sections. This model includes one additional signal input pin, labelled $P n S F R E Q$, that inputs an external voltage whose value is equivalent to the circuit simulation frequency. The test bench in Fig. 8 (a) gives the details of how the pin PnSFREQ voltage is generated using the "Parameter sweep" icon. Notice that in this test bench circuit the "Paramete sweep" icon sets the value of parameter $S F R E Q$ which in turn sets the value of component $V 1$, parameter freq, and component $I 1$, parameter $I$. In a similar 

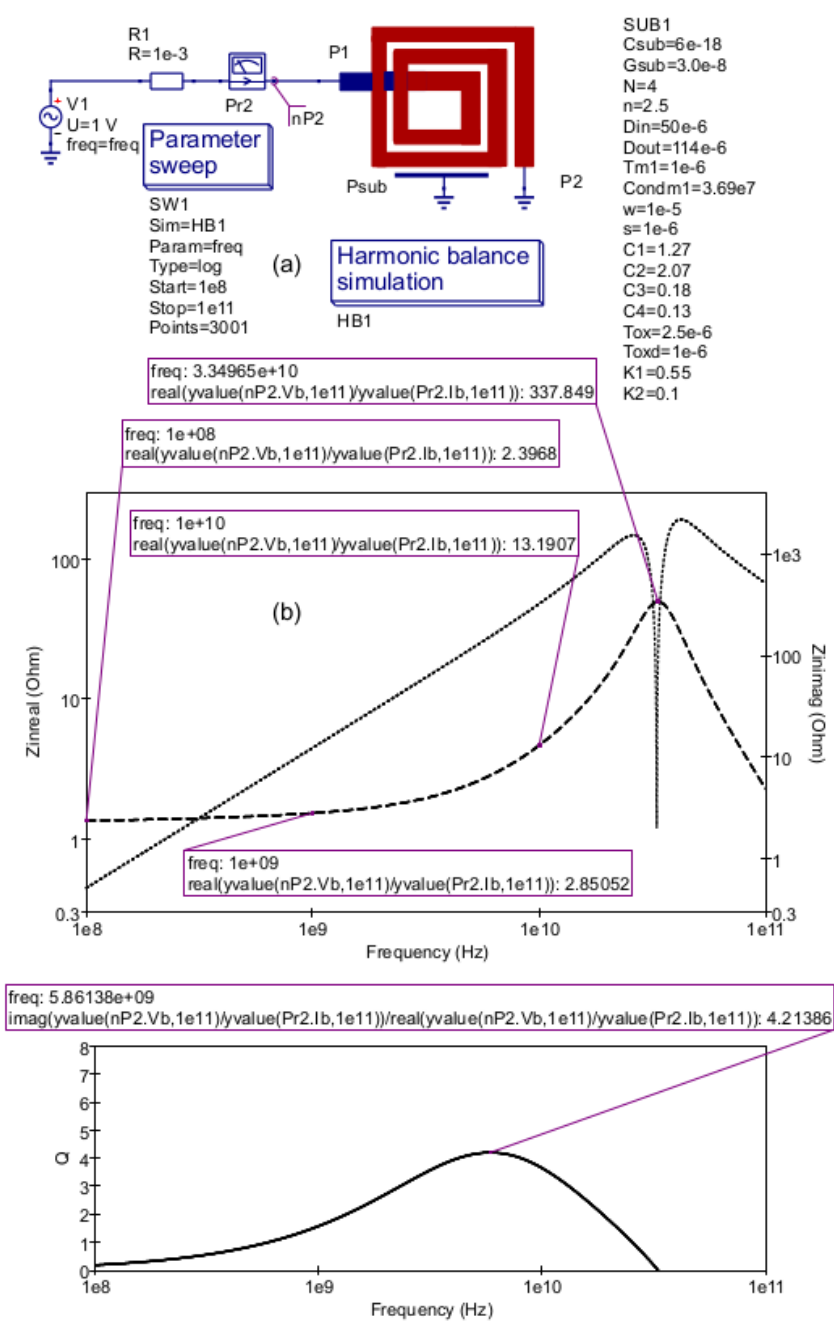

Fig. 6. Qucs and QucsStudio test bench and typical simulation output data with $R s(f)$ represented by an embedded FEDD: (a) Harmonic Balance test circuit, (b) Zinreal (dash curve) and Zinimag $(f)$ (dot curve) plotted against frequency, and (c) Inductor effective $Q$ factor plotted against frequency.

fashion to Fig. 6 the frequency of $\mathrm{HB}$ simulation, in Fig. 8 is set by $S F R E Q$. As far as the author is aware this indirect method of user controlled HB simulation has not been reported previously in the published literature.

\section{COMPARISON OF MEASURED AND SIMULATED SPIRAL INDUCTOR PERFORMANCE}

The performance of a spiral inductor on silicon is often assessed by two circuit parameters. These are (1) effective quality factor $Q=Z$ inimag/Zinreal and (2) self-resonant frequency Fres. The higher that both quantities are the nearer to an ideal inductance performance the spiral inductor becomes.

\section{A. $Q$ factor}

Over the frequency band $0.1 \mathrm{GHz}$ to $10 \mathrm{GHz}$ the recorded value of the simulated imaginary component of input impedance appears to be very similar across test benches. Values for Zinreal suggest a very different result where

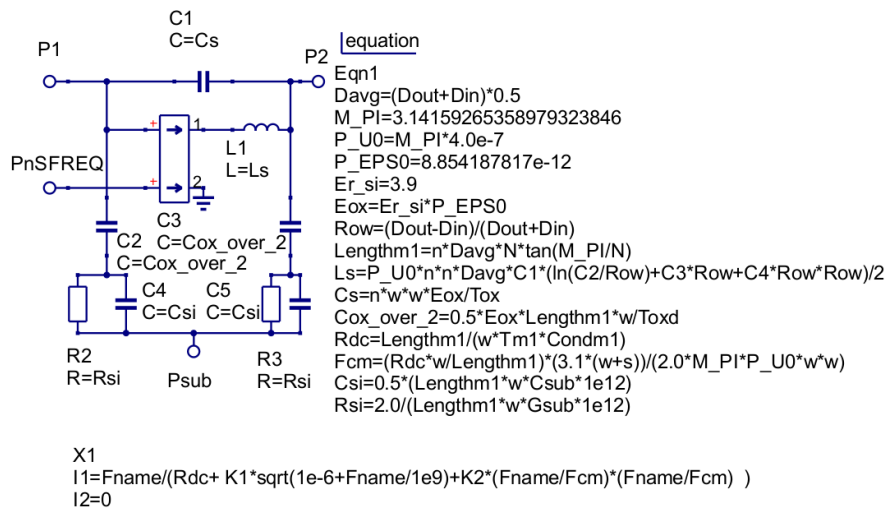

Fig. 7. An equivalent electric circuit for a spiral inductor with an embedded EDD representing $R s(f)$ : with Qucs Fname is written as $V 2$, with QucsStudio Fname is written as $-v 2$.

adding the $R \operatorname{skin}(f)$ and $R e d d y(f)$ components significantly increases Zinreal, particularly at higher frequencies, causing a reduction in effective Q. Summaries of the Zinreal and Q data are listed in Table I.

\section{B. Fres}

Measured and simulated values for the self-resonance frequency Fres are given in Table I and Table 2 II. The models where $R s=R s(f)$ indicated a more realistic comparison between the simulated and measured Fres data when compared to the case where $R s=R d c$. One other comment is worth making regarding the overall performance of the new simulation techniques introduced in this paper, namely that modelling using FEDD and EDD technology coupled with $\mathrm{AC}$ and $\mathrm{HB}$ analysis yields very similar results for the $Q$ and Fres circuit performance parameters. This observation implies that explicit and implicit user defined frequency/component parameter scans are viable as circuit simulation tools, adding a new tool for investigative Qucs/Qucs-S and QucsStudio compact modelling and simulation.

TABLE I

SPIRAL INDUCTOR SIMULATED CIRCUIT PARAMETER VALUES

\begin{tabular}{|c|c|c|c|c|c|c|c|}
\hline \multirow[t]{2}{*}{$R s$} & \multirow[t]{2}{*}{ Sim } & \multicolumn{3}{|c|}{$\begin{array}{c}\text { Zreal } \\
\Omega\end{array}$} & \multicolumn{2}{|l|}{$\mathbf{Q}$} & Fres \\
\hline & & $0.1 \mathrm{GHz}$ & $1 \mathrm{GHz}$ & $10 \mathrm{GHz}$ & $\max$ & $\mathrm{GHz}$ & $\mathrm{GHz}$ \\
\hline Rdc & $\mathrm{AC}$ & 2.22 & 2.23 & 2.78 & 22.41 & 1.803 & 38.10 \\
\hline FEDD & $\mathrm{AC}$ & 2.40 & 2.86 & 13.22 & 4.19 & 6.545 & 33.55 \\
\hline FEDD & $\mathrm{HB}$ & 2.40 & 2.85 & 13.19 & 4.21 & 5.861 & 33.50 \\
\hline EDD & $\mathrm{HB}$ & 2.40 & 2.85 & 13.22 & 4.22 & 5.859 & 33.54 \\
\hline
\end{tabular}

\section{CONCLUSION}

With the Qucs/Qucs-S and QucsStudio circuit simulators reaching a stage in their development where they offer mature compact modelling and simulation capabilities their use by the modelling and circuit design communities is beginning to identify new modelling and simulation techniques which were not obvious previously. Compact modelling of nonlinear frequency dependent components and advanced forms 

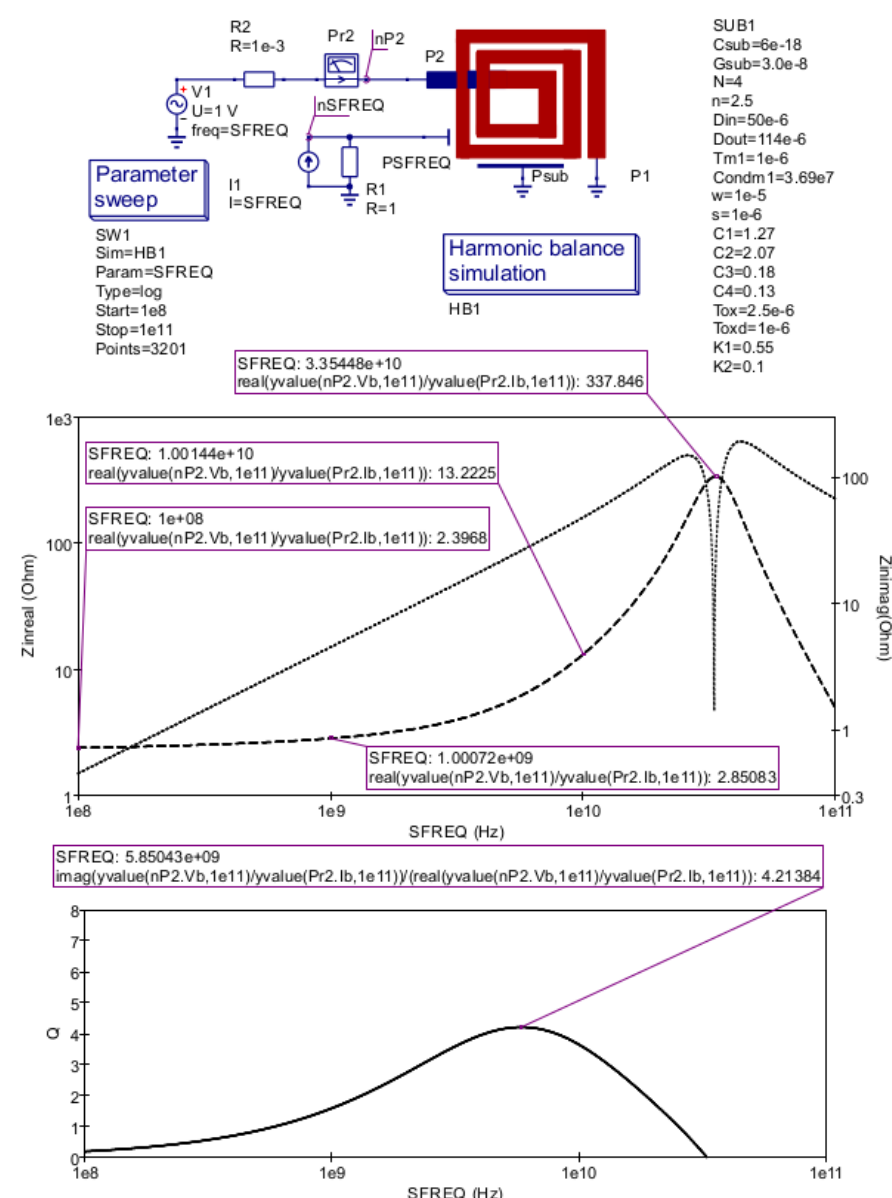

Fig. 8. Qucs and QucsStudio test bench and typical simulation output data with $R s(f)$ represented by an embedded EDD: (a) Harmonic Balance test circuit, (b) Zinreal (dash curve) and Zinimag(f) (dot curve) plotted against frequency, and (c) Inductor effective $Q$ factor plotted against frequency.

TABLE II

MEASURED AND SIMULATED SPIRAL INDUCTOR DATA

\begin{tabular}{|l|c|c|}
\hline Component & Measured values [16] & $\begin{array}{c}\text { Calculated or } \\
\text { simulated values } \\
\text { See Table I }\end{array}$ \\
Fres $(\mathrm{GHz})$ & 31.3 & 50.0 \\
Din $(\mu \mathrm{m})$ & 50.0 & 114 \\
Dout $(\mu \mathrm{m})$ & 114 & $3.45 \mathrm{e}-15$ \\
Cs $(\mathrm{F})$ & $6.14 \mathrm{e}-22$ & $2.82 \mathrm{e}-13$ \\
Cox $(\mathrm{F})$ & & $4.92 \mathrm{e}-14$ \\
Csi $(\mathrm{f})$ & & $8.13 \mathrm{e} 3$ \\
Rsi $(\Omega)$ & & 2.22 \\
Rdc $(\Omega$ & 2.75 & \\
Csub1 $(\mathrm{F})$ & $4.47 \mathrm{e}-13$ & \\
Csub2 $(\mathrm{F})$ & $3.33 \mathrm{e}-14$ & \\
Rsub1 $(\Omega)$ & 11.34 & \\
Rsub2 $(\Omega)$ & 4999 & \\
L $(\mathrm{H})$ & $7.68 \mathrm{e}-10$ & \\
Ls $(\mathrm{H})$ & & \\
\hline
\end{tabular}

of indirect user controlled swept HB circuit simulation are two examples of emerging features from multi-simulator and compact device modelling software packages. This paper introduces the background, principles and application of an unusual and advanced HB simulation technique through the development of a wide band microwave spiral inductor on silicon. Finally, the performance of the simulation model is compared with a set of previously published measured data for a similar device. Overall, very good correlation between the simulated and measured data was observed.

\section{REFERENCES}

[1] B. Johnson, T. Quarles, A.R. Newton, D. O. Pederson, A. SangiovanniVincentelli, "SPICE3 Version 3f User's Manual", Department of Electrical Engineering and Computer Sciences, University of California: Berkeley, CA. 1992.

[2] H. A. Wheeler, "Formulas for the skin effect," Proceedings of I.R.E. vol. 30, pp. 412-424,, Sept. 1942.

[3] M.E. Brinson and S.Jahn, "Modelling of high-frequency inductance with Qucs non-linear radio frequency equation defined devices," International Journal of Electronics, vol. 96, No. 3,March 2009, 3070321.

[4] S. Jahn, and M. Brinson, "Interactive Compact Modeling Using Qucs Equation-Defined Devices”, Int. J. Numer. Model. 2008, vol 21, pp. 335349

[5] Sourceforge, Qucs project: Quite Universal Circuit Simulator, Version 0.0.19. qucs.sourceforge.net. 2017. [Accessed February 2018].

[6] M. Margraf, QucsStudio Version 2.4 .1 , http://dd6um.darc.de/QucsStudio/qucsstudio.html, 2015, [Accessed February 2018].

[7] Sourceforge, "Ngspice: mixed-level/mixed-signal circuit simulator based on Berkeley's SPICE 3f5", Version 27, 2018. [Accessed February 2018].

[8] Sandia National Laboratories, "Xyce Parallel electronic simulator: version 6.8“, 2015, https://xyce.sandia.gov/ [Accessed February 2018].

[9] SPICE OPUS, "SPICE OPUS: Analog circuit simulation specially suited for optimization tools, based on SPICE $3 \mathrm{f} 5$ and XSPICE. Faculty of Electrical Engineering at the University, Slovenia. Version 2.3.2, https://spiceopus.si, 2018, [Accessed 2018].

[10] V. Kusnetsov and M. Brinson, "Qucs-S: Qucs with SPICE". Version 0.0.20, https://ra3xdh.github.io/, 2018. [Accessed 2018]

[11] C. P. Yue, C. Ryu, J. Lau, T.H Lee and S.S. Wong, "A physical model for planar spiral inductors on silicon", IEEE Electron Devices Meeting IEDM '96, San Fransisco CA, USA, 1996.

[12] J. Gil and H.Shin, "A simple wide-band on-chip inductor model for silicon-based RF ICs", IEEE Transactions on Microwave Theory and Techniques, vol. 51, No. 9, 2003, pp. 2013-2018.

[13] S.S Mohan, M.del Mar Hershenson, S.P Boyd and T.H. Lee, "Simple accurate expressions for planar spiral inductances", IEEE Journal of Solid-State Circuits, Vol. 34, No. 10 October 1999, pp. 1419-1424.

[14] W.B. Kuhn, X.He and M. Mojarradi, "Modeling spiral inductors in SOS processes", IEEE Transactions on Electron Devices, Vol. 51. No. 5, 2004 , pp. 677-6782.

[15] Kok-Yan Lee, S. Mohammadi, P.K. Bhatacharya and L.P.B. Katchi, ”A wideband compact model for integrated inductors", IEEE Microwave and Wireless Components Letters, Vol. 16, No. 9, 2006, pp. 490-492.

[16] N. Vashisht,"RF modeling of pasive components of an advanced submicron CMOS technology", San Jose State University, 2008.

[17] J. Burghartz and B. Rejaei, "On the design of RF Spiral Inductors on Silicon", TED, Vol. 50, No. 3, 2003. 